Rev. Col. Ciencia. Vol. 2, no. 1. Octubre 2020 - marzo 2021. ISSN L 2710-7434. pp. 15-24

\title{
LA LINGÜÍSTICA Y LA PRODUCCIÓN DEL TEXTO ESCRITO EN LOS ESPACIOS VIRTUALES
}

\author{
The linguistics and production of the written text in virtual spaces \\ Franklin Esteban Jaén \\ Universidad de Panamá, Centro Regional Universitario de San Miguelito, Facultad de \\ Humanidades. Panamá \\ franklinjaen-12@hotmail.com \\ https://orcid.org/0000-0001-8032-3405
}

\begin{abstract}
RESUMEN
En este artículo se analiza el significado de la lingüística y la producción del texto escrito en los espacios virtuales. Es una investigación documental, con diseño bibliográfico, referido al paradigma cualitativo, de carácter analítico-interpretativo. El método utilizado fue el hermenéutico, las técnicas de recolección de información: observación documental y resumen analítico, instrumentos: fichas, dispositivos electrónicos y matriz de análisis; análisis de datos: circulo hermenéutico de Guba y Lincoln, posteriormente, los datos fueron categorizados facilitando el proceso de teorización. Como consideraciones finales se plantea que se han incorporado códigos que han impactado la lingüística en la producción escrita que se desarrolla en los espacios virtuales tales como internet, chat, facebook, entre otros. Estas modificaciones son evidentes resaltando que se ha perdido la utilización de las vocales, la acentuación, los signos de puntuación con una transformación del lenguaje en los espacios que permiten la escritura en este paradigma de las tecnologías de la información y comunicación.
\end{abstract}

PALABRAS CLAVES. Lingüística, texto escrito, espacios virtuales, lenguaje, ciberlenguaje.

\begin{abstract}
This article discusses the meaning of linguistics and the production of text written in virtual spaces. It is a documentary research, with bibliographic design, referring to the qualitative paradigm, of an analytical-interpretive character. The method used was hermeneutics, information collection techniques: documentary observation and analytical summary, instruments: tabs, electronic devices and analysis matrix; data analysis: Guba and Lincoln hermeneutic circle, subsequently, the data were categorized facilitating the theorization process. As final considerations it is proposed that codes have been incorporated that have impacted linguistics on the written production that takes place in virtual spaces such as internet, chat, facebook, among others. These modifications are evident highlighting that the use of vowels, accentuation, punctuation have been lost with a transformation of language in spaces that allow writing in this paradigm of information and communication technologies has been lost.

KEYWORDS. Linguistics, written text, virtual spaces, language, cyberlanguage.
\end{abstract}

Artículo recibido: 23 de agosto de 2020

Artículo aceptado: 14 de septiembre de 2020 


\section{INTRODUCCIÓN}

El avance de las tecnologías de la información y la comunicación (TIC), a nivel mundial, ha impactado en diferentes escenarios tanto sociales, económicos, políticos como educativos. Así, los procesos del lenguaje y, en particular de la escritura, se han visto notoriamente influenciados por el fenómeno de la virtualidad, tanto en el desarrollo de las competencias inherentes a este, como en la producción, difusión y recepción de los textos. Por consiguiente, se dinamizan una serie de operaciones sociocognitivas para el acceso, la transformación, generación y socialización de este conocimiento. Entendiendo, que para concretizar la dimensión textual escrita no solamente se depende de la apropiación de los nuevos medios y del nivel de literacidad electrónica, sino también del dominio de la lengua, la comunicación, los géneros discursivos y tipo de texto. (Carvajal López, 2015, p.12 ). Desde esta perspectiva la producción escrita implica la creación de ambientes y herramientas que propicien la construcción, la manipulación, circulación eficiente de los textos y, la creación de comunidades de aprendizaje mediante la interacción social.

Por otra parte, el texto escrito, está condicionado por el universo del discurso, es decir, por el sistema ecuménico de significaciones al que pertenece determinando así su validez y sentido. De allí, resulta importante entender que la lingüística textual escrita no solo debe considerarse como un conjunto de frases y párrafos con determinada estructuración, sino que visualiza al texto como la unidad fundamental del lenguaje con sentido comunicativo (Carvajal López, 2015, p.21 ). Es decir, la distribución de los enunciados que forman el texto escrito está en relación con los temas, subtemas y cambios que se dan en interdependencia con el contexto. Además, debe atender a los elementos que se vincula permitiendo la coherencia de los enunciados, según D’Sousa Carmet, (2015), son parte de éstos "la ortografía; puntuación; uso de preposiciones, adverbios y artículos definidos e indefinidos; el empleo de los pronombres relativos; las concordancias gramaticales; el uso de la subordinación sintáctica y la propiedad léxica, todo ello dando coherencia local al texto" (p. 34). En consecuencia, se genera sentido y cohesión en la secuencia de proposiciones organizadas sintáctica y semánticamente con propósitos comunicativos.

Sobre esto resalta Mongada Ostios (2015) que "los sistemas de escritura proporcionan un modelo para la lengua y el pensamiento, por lo que, la conciencia de la estructura lingüística es un producto de esta, no una condición previa a su desarrollo" (p. 33). Se entiende que, no hay un solo tipo de cultura escrita, sus usos y diferentes grados de importancia se circunscriben al contexto en el que se desarrolla por lo que la producción textual escrita obedece a propósitos comunicativos y lingüísticos diversos.

En cuanto a los entornos virtuales, estos representan espacios de comunicación abiertos de interconexión a través de ordenadores o dispositivos, así como de memorias informáticas condicionando la producción escrita, ya que, existe una relación entre esta práctica y el soporte en el cual se subscribe, generando una sinergia en la que el escritor interactúa alternativamente como emisor y receptor, haciendo uso del lenguaje como tal y, de las habilidades comunicativas. De lo anterior indica Carvajal López (2015) que:

La escritura asociada a la tecnología de la comunicación comprende dos dimensiones: la material, constituida por la herramienta, los aparatos, la conectividad, los sistemas de información y, otra inmaterial, representada por los conocimientos que la produjeron y aquellos necesarios para su uso y funcionamiento, ambas permeándose mutuamente. (p. 44) 
Por consiguiente, el propósito de la producción escrita opera desde una lógica propia e inexorable que deviene de manera incontrolable de los espacios y herramientas virtuales, enmarcados en una dinámica sociocultural asociada a la práctica y uso específico.

Se tiene a Riascos Diaz (2015) en su trabajo titulado "El lenguaje y cibertexto", una investigación de tipo documental, indica que:

La categoría denominada cibertexto, es entendida como el discurso generado en espacios virtuales en el que confluyen el texto, la imagen, el sonido y, la infografía; todos como elementos que se articulan para dar paso a una unidad con sentido completo, no lineal, estructurada con enlaces y que posee una marcada esencia social al ser completada por el propio usuario/lector quien busca la información a la medida de sus necesidades e intereses (p.35).

Se entiende que, en este ciberlenguaje como forma de propiciar la producción escrita, se presenta una variabilidad de la gestión linguiística en función de la forma y modos de presentación del discurso escrito. Surgiendo una adecuación del lenguaje, del género discursivo y de otros rasgos lingüísticos de acuerdo con el contexto en el que se produzca.

Por otra parte, Mujica Santos (2017) en su investigación denominada "El modelo contextual interaccionista y las estrategias metalingüísticas en el cibertexto" estudio de carácter descriptivo que permitió presentar las principales características del cibertexto dentro de la producción escrita virtual indicando que estas corresponden a:

- La multimedialidad, entendida como la capacidad de procesar y difundir mensajes que integran diversos códigos linguísticos textuales, visuales y sonoros que gozan de unidad de contenido comunicativa, evidenciando la retórica del lenguaje y convirtiéndose en signos eficaces de transmisión siempre que sean adecuadamente utilizados. Esta propiedad es equivalente a la coherencia y cohesión en la escritura análoga impresa.

- La interactividad, recupera la dimensión participativa entre el escritor y el lector, la producción escrita se convierte en un diálogo, permitiendo que la visión del texto escrito evolucione en un espacio donde la información cuenta con una multiplicidad de formas en la que puede presentarse logrando varios sentidos en su construcción y decodificación.

- Hipertextualidad; entendida como un sistema de organización y presentación del texto escrito, basada en la vinculación de documentos o fragmentos de éstos (textuales o gráficos) en cualquier morfología digital: texto, imagen, audio, vídeo y, otras aplicaciones capaces de generar un contenido dinámico, lo que permite acceder a la información no necesariamente de forma secuencial. (p. 47)

De esta manera, las transformaciones en los modos de escribir a partir de la confluencia en el espacio virtual orientan a plantear su significado, identificando los conocimientos procedimentales de la cultura escrita desde la dimensión lingüística; entendiendo que ésta última hace referencia a la naturaleza misma del enunciado, es decir, a su estructura superficial, la cual es configurada por relaciones sintácticas y semánticas que rigen su producción. (Malavé Enriquez, 2016, p. 33 ) 
Rev. Col. Ciencia. Vol. 2, no. 1. Octubre 2020 - marzo 2021. ISNN L 2710-7434. pp. 15-25

En cuanto a las técnicas para presentar la producción escrita en espacios virtuales Ponte Villareal (2016), en su trabajo titulado "El discurso escrito digital", investigación de carácter descriptivo, analiza las más relevantes

1. Claridad: Esto significa precisión y nitidez, debe explicar claramente el mensaje o ideas centrales de la información, por lo que se requiere evitar el lenguaje relativo, complejo o con excesiva tecnicidad. Se recalca la forma de presentación sencilla y progresiva del contenido para mantener la atención e interés del lector.

2. Extensión: Evitar la sobrecarga informativa, utilizando como máximo 8 líneas por párrafo. Se debe mantener parámetros en el manejo del contenido escrito para orientar el mensaje.

3. Estructura: El contenido debe estar guiado por una lógica la cual facilite y oriente la navegación a través de enlaces y dentro del mismo texto. Además, esta organización fomenta el desarrollo de procesos lógicos del pensamiento del lector, a través de un texto principal del contenido, seguido por la presencia de ideas claves, fragmentos aclaratorios, entre otros y, un bloque textual de síntesis que recoge las ideas centrales en forma resumida.

4. Elementos lingüísticos: El ciberespacio posee una marcada división lingüística. Se debe entender que las modificaciones encontradas ocurren principalmente por dos motivos: creatividad del escritor y la necesidad de rápida comunicación, por lo que organizar el contenido dentro de un texto, considerando la dimensión lingüística en su totalidad, es un punto de cuidado y atención, aunque parezca evidente, esta consideración, en oportunidades, se pasa por alto lo que perjudica que un contenido de valor alcance al final los objetivos deseados. La red ofrece un nuevo entorno para el lenguaje, más dinámico que el discurso escrito tradicional (p. 44).

De esta manera, el texto escrito en los espacios virtuales requiere de ciertas condiciones que le permitan ser lo más conversacional posible, establecer la empatía adecuada de forma que el lector no se pierda en lo que se expresa. La claridad, el manejo adecuado de las incidentales y sintaxis lineal como herramientas infaltables; asimismo la brevedad con contenido y la explicación sin fatiga. Para ello, se requiere cuidar el exceso de sustantivos y adjetivos, además de las expresiones ambiguas. Todo esto contribuye a una producción escrita atractiva y efectiva en el escenario de la virtualidad.

No obstante, a pesar de existir autores y literatura a favor del tema de la producción del texto escrito en los espacios virtuales, se encuentran de igual manera, otros detractores que expresan que el estilo de producción lingüística escrita rápida que caracteriza a la virtualidad ha generado el empleo de diversas e intencionadas modalidades de escritura o heterografías que en general deforman el lenguaje. Así lo señala el estudio titulado "La conciencia de la estructura lingüística escrita en la virtualidad", investigación descriptiva que concluye que la producción textual escrita en los diferentes espacios virtuales, tales como: correos electrónicos, la world wide web, chats, blogs, mensajes instantáneos, whassap y mucho más, desdibujan el ámbito lingüístico en su configuración y, en algunos casos, pasan a tener menor consistencia, interesándose el lector más por el significado del contenido que por la dimensión pragmática del lenguaje como tal, estas presencias difusas son: el carácter ortográfico, sintáctico, textualidad, estructuración del texto, dificultades de estilo y vocabulario. (Mongada Ostios, 2015, p.32) 
Rev. Col. Ciencia. Vol. 2, no. 1. Octubre 2020 - marzo 2021. ISNN L 2710-7434. pp. 15-25

Indica Terrado Cárdenas (2015) en su trabajo titulado "El español en el ciberespacio: Creación y cambios culturales" que:

En la producción textual escrita el lenguaje digital cuenta con una sintaxis sumamente compleja y poderosa, pero carece de una semántica adecuada en el campo de la relación. Priva la economía de las palabras, y la urgencia en hacerlas llegar pronto al destinatario. [...] La mayoría de los textos de las redes sociales, por el contrario, son breves, espontáneos, cortos y poco meditados. Es el triunfo de la inmediatez comunicativa frente a la reflexión intelectual. Es el triunfo de la escritura del SMS y, no del texto narrativo. (p. 77).

Se afirma que, la producción textual escrita digitalmente desdibuja el sentido lingüístico, esto por la brevedad del mensaje, no siguen las reglas ortográficas ni de redacción, utilizan acrónimos, elementos y abreviaturas no gramaticales, solo por similitud fonética o signos no fonéticos como los emoticones; se observa una mayor informalidad y proximidad con el habla que las características de la escritura formal análoga o impresa.

Con base a lo planteado, surge el interés de estudio que tiene como objetivo analizar el significado de la lingüística y la producción del texto escrito en los espacios virtuales, entendiendo que es una actividad compleja en la cual se requiere la organización jerárquica de los contenidos semánticos para lograr transcribirlos en una ordenación lingüística no lineal, pero que, sin embargo, plantea a los usuarios de la escritura nuevos retos, exigencias y posibilidades.

\section{MATERIALES Y MÉTODOS}

El componente metodológico de la investigación remite a la aplicación del paradigma cualitativo, atendiendo a lo indicado por (Martínez, 2015) corresponde a "una actividad sistemática orientada a la comprensión de fenómenos mediante el estudio interpretativo de realidades, coadyuvando a la comprensión interpretativa y comprensiva de esta abordada desde diferentes escenarios, posibilitando efectuar distintas lecturas buscando el sentido y significado contextuales" (p.45). Por otra parte, se fundamentó en una investigación teórica de tipo documental con diseño bibliográfico, de carácter analítico e interpretativo-crítico, definida por Méndez (2015) como "estudios de desarrollo teórico presentando a partir del análisis crítico de la información empírica y teorías existentes" (p. 7). El abordaje y logro de los objetivos se hizo, fundamentalmente, desde un proceso hermenéutico, incluyendo las fases de descripción, análisis e interpretación teórica.

El procedimiento de la investigación atendió al esquema planteado por Véliz Angulo (2016), para los estudios documentales, efectuado de acuerdo con los siguientes pasos:

1. Ubicación y arqueo de fuentes bibliográficas y documentales: textos, informes, proyectos, foros, seminarios, artículos de revisas, entre otros, que permitieran obtener la información pertinente del objeto de estudio.

2. Selección de las fuentes apropiadas para recoger datos relevantes.

3. Validación de las fuentes seleccionadas.

4. Lectura detallada de las fuentes seleccionadas, relacionada con los eventos de estudio.

5. Localización y fichero del material relevante.

6. Codificación y sistematización de la información seleccionada con el registro de esta en el análisis acorde a sus contenidos.

7. Establecimiento y descripción de las categorías de los eventos objeto de estudio para los cuales se pretende identificar relaciones. 
Rev. Col. Ciencia. Vol. 2, no. 1. Octubre 2020 - marzo 2021. ISNN L 2710-7434. pp. 15-25

9. Análisis comparativo de los datos aportados por las teorías seleccionadas, en relación con los eventos de estudio, a través de la clasificación y descripción de estos.

10. Conclusiones a partir del análisis de los datos obtenidos, para responder al objetivo general (p. 55).

En cuanto a las fuentes de información se consideraron las secundarias, referidas como todo soporte material, tales como documentos escritos, libros, periódicos, revistas, actas notariales, tratados, encuestas, conferencias escritas, documentos fílmicos y digitales que aportan datos del objeto de estudio (Arias, 2016, p. 24).

Para el análisis de las fuentes documentales se emplearon como técnicas: La observación documental, presentación resumida de texto, resumen analítico. Además de las operacionales: Subrayado, fichaje, citas y notas bibliográficas, presentación de cuadros y gráficos. En cuanto a los instrumentos se seleccionaron: (a) Fichas; (b) Computadoras y sus unidades de almacenajes; (c) Cuadros de registros y clasificación de categorías; y (d) la Matriz de Análisis, a través de esta se extrajo la información de los documentos revisados que fundamentan este estudio.

Con referencia al procesamiento y análisis de la información, se utilizó el análisis de contenido y el círculo hermenéutico-dialéctico referido por Lincoln y Guba. Posteriormente, se definieron las categorías de análisis para la consiguiente teorización. Las categorías de análisis quedaron expresadas: (1) El texto escrito en los espacios virtuales; (2) Dimensión lingüística para la producción textual escrita en espacios virtuales; (3) Técnicas para presentar la producción escrita en espacios virtuales y; (4) Situaciones frecuentes de presencia difusa lingüística en la producción textual escrita virtual.

Los argumentos esgrimidos fueron confrontados e interpretados hasta lograr un entendimiento y consenso, respetando los diferentes puntos de vista y teorías. Particularmente y, con el fin de garantizar la validez, la información construida documenta fehacientemente las relaciones particulares existentes; la validez y confiabilidad a través del método hermenéutico de investigación, se obtuvo en los términos, de validez de contenido: El entendimiento del discurso condujo a construir e interpretar mancomunadamente argumentos y acuerdos para la comprensión plena; validez cognitiva: consistió en la viabilidad para evidenciar el esfuerzo por el desarrollo de la capacidad creadora y los procesos subjetivos de pensamiento y reflexión acuerdo a la teoría de (Véliz Angulo, 2016, p.77). De esta manera, se logró ajustarlo a lo que se requiere en lo explicativos y validez de interpretación haciendo énfasis en el parafraseo y postura personal del investigador en función del análisis de los referentes expuestos por los diferentes autores consultados.

\section{RESULTADOS}

Los treinta y cuatro (34) documentos analizados se clasificaron en una secuencia cronológica, resaltando tópicos en cuanto aportaciones teóricas presentadas por cada uno, enfatizando las argumentaciones dadas en los conceptos, teorías y lenguajes propios de la disciplina en la que se ha formado el autor que hace la contribución. Se configuro la matriz de análisis con cuatro componentes, según las categorías definidas a saber:

\section{EI Texto escrito en los espacios virtuales}


La idea central se enfoca en entender que la producción escrita implica la transformación de los conocimientos individuales del ser, sin embargo, con la llegada del ciberespacio, las redes sociales, entre otros espacios virtuales, se plantea a los usuarios de la escritura nuevos retos, exigencias y posibilidades.

Se tiene que, en el medio digital el texto escrito es el punto de entrada para los lectores. El bloque visual es lo que primero atrae la mirada del usuario. Por lo que la extensión es importante, se requiere segmentar la información en párrafos cortos que sean fáciles de leer que no superen las cinco o seis líneas, utilizando otras herramientas para profundizar el detalle del texto, tal como el hipertexto, entre otros.

\section{Dimensión linguiística para la producción textual escrita en espacios virtuales}

Las transformaciones en los modos de escribir a partir de la confluencia en el espacio virtual orientan a plantear su significado, identificando los conocimientos procedimentales de la cultura escrita desde la dimensión lingüística; entendiendo que ésta última hace referencia a la naturaleza misma del enunciado, es decir, a su estructura superficial, la cual es configurada por relaciones sintácticas y semánticas que rigen su producción.

La producción textual escrita digitalmente desdibuja el sentido lingüístico, esto por la brevedad del mensaje, no siguen las reglas ortográficas ni de redacción, utilizan acrónimos, elementos y abreviaturas no gramaticales, solo por similitud fonética o signos no fonéticos como los emoticones; se observa una mayor informalidad y proximidad con el habla que las características de la escritura formal análoga o impresa.

Se evidenció que el texto escrito virtual ya no se construye solo con oraciones, párrafos y reflexiones de manera lineal sobre un tema determinado, sino que, además, la tecnología digital posibilita la generación de ésta con otros lenguajes, como el visual y el auditivo que ofrecen una nueva dimensión desde la integralidad de la virtualidad, esta forma discursiva se le denomina competencia multimodal.

En el espacio virtual se escribe para un público pluricultural y plurilingüístico, la escritura, las inconsistencias en la escritura no se consideran como un fallo lingüístico sino de pensamiento, dándole mayor significancia a la claridad y alcance del mensaje.

\section{Técnicas para presentar la producción escrita en espacios virtuales}

La virtualidad confiere la multiplicidad de recursos que facilitan empoderar las habilidades lingüísticas y sintácticas para la estructuración del discurso escrito haciéndolo accesible en el sentido comunicativo y de procesamiento multidimensional del texto, por lo que se requiere: Claridad, extensión estructura elementos lingüísticos.

\section{Situaciones frecuentes de presencia difusa lingüística en la producción textual escrita virtual}

Estas presencias difusas son:

- De carácter ortográfico: Ausencia de marcación de tildes y adecuada acentuación; inadecuada escritura de algunas letras como la s, c, z, b, v, entre otras; escaso uso de signos de puntuación sin considerar el sentido y la intencionalidad 
del escritor y las reglas de uso de estos; también se presentan errores de digitación; de igual modo, se evidencian acortamientos léxicos no estandarizados; el uso inadecuado o carencia de las mayúsculas cuando la norma así lo establece.

- De carácter sintáctico: construcción inadecuada de oraciones.

- Sobre la textualidad: Falta de cohesión, se evidencia poco uso de conectores que articulen adecuadamente palabras, oraciones o párrafos entre sí. Fallas de coherencia y relación entre las ideas.

- Estructuración del texto: No hay introducción de la temática, ni desarrollo adecuado de las ideas, así como conclusión del escrito.

- Escasa planificación del texto por escribir y, poca revisión antes de su difusión.

- Dificultades de estilo y vocabulario en la micro redacción, en algunos casos tendiendo a oralizarse, presentan rasgos de coloquialismo. Problemas de macro redacción, se evidencia en algunos escritos falta de organización de la información de acuerdo con un sentido lógico.

- Las variantes de lengua escrita (legal, religiosa, entre otras) pueden encontrarse con escasos cambios estilísticos, aparte de los que exige la adaptación a un medio electrónico. Por el contrario, las situaciones de correo electrónico, chats, mundos virtuales y mensajería instantánea, aunque expresadas en escritura, manifiestan varias características propias del habla. Están gobernadas por el tiempo, esperan o exigen respuesta inmediata, son transitorias, en el sentido de que pueden ser borradas inmediatamente (como en los e-mails) u olvidarse conforme desaparecen de la pantalla (como en los chats) y, sus manifestaciones poseen mucho de la urgencia y la energía propias de las conversaciones cara a cara.

- La red permite el mismo grado de planificación y complejidad linguística estructural que puede encontrarse en textos escritos e impresos en papel. Pero en los chats, los mundos virtuales y la mensajería instantánea, donde existe una gran presión por comunicarse con rapidez, hay mucha menos complejidad y planificación lingüística.

\section{DISCUSIÓN}

Desde el análisis e interpretación de las diferentes fuentes secundarias consultadas sobre el tema en la discusión de los resultados se precisa:

Como parte de los conocimientos procedimentales vinculados a la producción escrita en el ciberespacio se evidencia el impacto en la dimensión lingüística, encontrándose la generación de textos, de acuerdo al espacio virtual, con prevalencia de mensajes y oraciones fragmentadas, empleo de gran cantidad de abreviaturas, introducción de enlaces, uso de emoticones y otras simbologías, la presencia de implícitos, el uso no canónico del alfabeto, el empleo de nuevos léxicos asociados a la cultura escrita en el ciberespacio, la presencia de imágenes, creación o adhesión a hashtag como representación de macroestructuras textuales, que de manera recurrente generan un desfase en la continuidad de los mensajes, entre otros. Esto se vincula con lo referido por Sanabria Torres (2015), afirmando que "los espacios virtuales influyen de manera notoria en la escritura, debido a que no tiene, en determinados contextos, un registro tan formal, sino que presentan un carácter más flexible y espontáneo" (p.44). De esta manera se visiona una nueva 
forma de comunicación que desde la perspectiva holística requiere ser entendida para configurar formas de socialización adaptadas a novedosas realidades lingüísticas.

Con respecto a la producción de textos en las redes sociales, tales como Facebook y Twitter, la revisión documental expone que la escritura trasciende, en oportunidades, los límites de temporalidad. Escribir en estos escenarios es un acto público que implica interactuar con otros, lo que ubica tal práctica en un lugar distinto al de la escritura análoga o impresa. Así mismo, prácticas marginales como el uso de apócopes y abreviaturas se convierten en tendencias generalizadas, constituyéndose en recursos funcionales que se erigen como un conocimiento procedimental necesaria en la escritura digital. Ciertamente, señala D’Sousa Carmet (2015) que "la virtualidad confiere la multiplicidad de recursos que facilitan empoderar las habilidades lingüísticas y sintácticas para la estructuración del discurso escrito haciéndolo accesible en el sentido comunicativo" (p. 56). Los medios electrónicos añaden nuevas posibilidades a las maneras en que las personas pueden manipular el texto escrito, creando nuevas formas de discurso electrónico y, por consiguiente, de aspectos lingüísticos en estos.

No obstante, en espacios formales virtuales como los académicos, de textos de jurisprudencias, escritura literaria o periodística, que mantienen propósitos formativos, se observa que se abrevian recursos reflexivos y prácticos para reconstruir las intervenciones en torno a la literacidad académica y laboral, sin embargo, se mantiene la dimensión linguiística. Por lo cual se infiere que desde la virtualidad, es posible fortalecer los procesos didácticos en torno a la escritura académica, empoderando el desarrollo de la creatividad y la capacidad crítica del escritor y lectores; esto resulta vinculante a lo expuesto por Riascos Diaz (2015), cuando "considera que ciberlenguaje como forma de propiciar la producción escrita, presenta una variabilidad de la gestión lingüística en función de la forma y modos de presentación del discurso escrito" (p. 33) Surgiendo una adecuación del lenguaje, del género discursivo y de otros rasgos linguiísticos de acuerdo con el contexto en el que se produzca. Los textos formales mantendrán su carácter de acuerdo a su naturaleza, no ocurriendo lo mismo en aquellos de carácter informales o impersonales.

\section{CONCLUSIONES}

Dentro de las consideraciones resaltantes se establecen:

1. La producción textual escrita presentes en los espacios virtuales de las redes sociales, tales como Facebook, Twitter y chat, entre otros, en cuanto a la dimensión linguística, se diferencia de las prácticas asociadas al manuscrito y al impreso, dado que involucran conocimientos procedimentales específicos que desplazan los conocimientos propios de la cultura escrita y, conceptos particulares sobre lo que significa escribir. Entre estos en el saber hacer de la cultura escritura virtual, se encuentran de manera difusa: el manejo de los signos de puntuación, la ortografía, el uso de preposiciones, adverbios, conectores y pronombres relativos, repetición de fonemas, mayúsculas sostenidas, las concordancias gramaticales, la propiedad lexical, la coherencia y cohesión local.

2. De igual manera, se presencia en la dicotomía lingüística formas expresivas no alfabéticas: emoticones, sticker, fotografías, audios, videos que confluyen como recursos propios de la cultura escrita virtual. Esta utilización de recursos no lingüísticos hace que la construcción de enunciados verbales tenga un peso menor en la representación de rasgos translingüísticos o de la fuerza ilocucionaria escrita. 
Rev. Col. Ciencia. Vol. 2, no. 1. Octubre 2020 - marzo 2021. ISNN L 2710-7434. pp. 15-25

3. Por otra parte, la multimodalidad textual deja de ser privilegio de especialistas para estar al alcance de los noveles escritores y comunidad general en los espacios virtuales.

\section{REFERENCIAS}

Arias, F. (2016). Metodología de la Investigación. Caracas, Venezuela: IMPM.

Carvajal López, A. (2015). Lengua y escritura en internet. Barcelona, España: Ariel Practicum.

D’Sousa Carmet, D. (2015). El ciberespañol: características del español usado en Internet. Sao Paulo, Brasil: Editora Hucitec.

Malavé Enriquez, C. (2016). La escritura y la informática. México: Siglo XXI Editores.

Martínez, A. (2015). Ciencia y arte de la investigación cualitativa. México D.F., México: Trillas.

Méndez, C. (2015). Metodología de la investigación. México: Mc GrawHill.

Mongada Ostios, B. (2015). La conciencia de la estructura linguistica escrita en la virtualidad. Madrid, España: E-ducativa.

Mujica Santos, C. (2017). El modelo contextual interaccionista y las estrategias metalingüísticas en el cibertexto. Aneca.

Nieto Alargón, M. (2015). Metodología de la Investigación. Bogotá. Colombia: Ediciones de la U.P.

Ponte Villareal, T. (2016). El discurso escrito digital. Tesis Doctoral. Caracas, Venezuela: Universidad Central de Venezuela, Facultad de Lingüística.

Riascos Diaz, E. (2015). Lenguaje y cibertexto. Madrid, España: Cambridge.

Sanabria Torres, C. (2015). Nuevos conocimientos, conceptos y prácticas de la cultura escrita en el ciberespacio. Tesis Maestria. Cali, Colombia: Universidad del Valle.

Terrado Cárdenas, L. (2015). El español en el ciberespacio: Creación y cambios culturales. Cartagena, Colombia: Fondo de Cultura.

Véliz Angulo, E. (2016). Fenomenología de la percepción. Barcelona, España: Planeta-Agostini. 